\title{
Local Event Forecasting and Synthesis Using Unpaired Deep Graph Translations
}

\author{
Yuyang Gao \\ George Mason University \\ Fairfax, VA \\ ygao13@gmu.edu
}

\author{
Xiaojie Guo \\ George Mason University \\ Fairfax, VA \\ xguo7@gmu.edu
}

\author{
Liang Zhao \\ George Mason University \\ Fairfax, VA \\ lzhao9@gmu.edu
}

\begin{abstract}
Local rare event forecasting and synthesis on networks are highly useful for emergence management. For example, synthesizing traffic congestion and disease diffusion over the road network and disease-contact network respectively of specific geo-locations is highly important for transportation planning and disease outbreaks intervention. This task requires to learn how the events of congestion or disease "translate" the graph patterns from source mode (e.g., without event) to target mode (e.g., with event) based on historical data for some locations. Then it needs to apply such "translation" upon a source-mode graph pattern in a new location's network, in order to estimate and foresee what it will look like in target-mode in this location.

Such task is called graph translation, which is an analogy and generalization to image and text translation. Similar to the situations in image and text translation, paired training data, which consists of pairs of source-mode graph and its corresponding targetmode, will usually not be available. In this work, we propose an approach for learn the translation of graphs from source-mode to target-mode such that the generated target-mode is indistinguishable from the distribution of the real target-mode using an adversarial loss. Because there is no paired training data, we also learn an inverse translation from target-mode to source-mode and couple these two translation mappings through cycle consistency loss. Extensive experiments on both synthetic and real-world application data demonstrate that the proposed approaches is capable of generating graphs close to real target graphs. Case studies on the synthesized networks have also been illustrated and analyzed to show the reasonableness of the generated target-mode graphs.
\end{abstract}

\section{CCS CONCEPTS}

- Computing methodologies $\rightarrow$ Neural networks;

\section{KEYWORDS}

Deep learning, representation learning, graph generation, graph translation

Permission to make digital or hard copies of all or part of this work for personal or classroom use is granted without fee provided that copies are not made or distributed for profit or commercial advantage and that copies bear this notice and the full citation on the first page. Copyrights for components of this work owned by others than ACM must be honored. Abstracting with credit is permitted. To copy otherwise, or republish, to post on servers or to redistribute to lists, requires prior specific permission and/or a fee. Request permissions from permissions@acm.org.

LENS'18, November 6, 2018, Seattle, WA, USA

(C) 2018 Association for Computing Machinery.

ACM ISBN 978-1-4503-6035-7/18/11.\$15.00

https://doi.org/10.1145/3282866.3282872
ACM Reference Format:

Yuyang Gao, Xiaojie Guo, and Liang Zhao. 2018. Local Event Forecasting and Synthesis Using Unpaired Deep Graph Translations. In 2nd ACM SIGSPATIAL Workshop on Analytics for Local Events and News (LENS'18), November 6, 2018, Seattle, WA, USA. ACM, New York, NY, USA, 8 pages. https://doi.org/10.1145/3282866.3282872

\section{INTRODUCTION}

Local societal events such as organized crimes, civil unrest, and disease outbreaks, have significant impacts on the society [46]. Forecasting, simulating, and synthesizing local events in specific geo-locations are highly important for infrastructure planning, emergence reaction, and disaster management. For example, it is important to forecast and synthesize the damage of the cities' networks of electrical power and communications due to a hurricane before it arrives, so that we can proactively enhance the infrastructure and optimize the logistics accordingly. This is especially important for those locations that do not have sufficient historical records on that type of disasters as they are highly vulnerable due to lack of corresponding experience. For example, the city of Atlanta is a southern city in the U.S. which seldom suffered from snow storm but on January 21, 2014 it had a big snow storm that easily paralyzed its transportation system because of scarcity of such experience in its history [11]. Similar for other types of events such as terrorism events: although most of the cities in the world have not experienced terrorism events, all of them would like to know how to handle it once it happens in the future, e.g., how to console the people and control the panics and rumors in social media and networks.

As exemplified above, local events usually can be formulated as patterns in networks and graphs which are universal representations of pairwise information in many problem domains such as social networks [43], transportation system [7], cyber network [26], and spatial regions [12]. To improve the performance of graphbased learning tasks, many recent research efforts have concentrated on encoding a graph (or graph nodes) into a vector representation, either through solving graph embedding tasks [16, 18, 35, 42] or developing graph neural networks by extending well-known network architectures (e.g., convolutional and recurrent neural networks) to graph data $[4,9,25,28,38]$. However, to forecast and synthesize the future potential local event patterns over graphs, we will need generative models for synthesizing graphs under some distributions.

Traditional generative models for graphs such as Barabasi-Albert model [1] and random graphs [10] are hand-engineered to model a particular family of graphs. But for complex local societal events, the distributions and types of the graphs are unknown and typically 
too sophisticated to be modeled by any single types of traditional graph generation models. For example, the Barabasi-Albert model is carefully designed to capture the scale-free nature of empirical degree distributions, but fails to capture many other aspects of real-world graphs, such as community structure. Indeed, for many real-world problems the real topologies of graphs are unknown, and hence we need end-to-end ways to infer the graph distributions from the graph observations.

Deep generative models is considered one of most successful methods to learn the coherent latent representations for automatically synthesizing and generating continuous data like images and audios. Recently, motivated by the applications such as chemical molecular designing or electrical circuit development [8], several attempts have been made for generating discrete-structured data such as graphs based on deep learning models. They are typically unconditional models which can only generate the similar graphs exactly following the original distributions of training data. However, due to spatial heterogeneity [39], in many situations such as local event forecasting and synthesis over graphs, we need conditional settings where the graph generation must condition on specific scenarios such as the local features. Most recently, there is one initial work [17] which can address the conditional graph generation, called graph translation, which is able to synthesize a target-mode graph in target domain by transforming from an source-mode graph in source domain. Specifically, from all the historical records of the pairs of source-mode graphs in source domain and their corresponding graphs in target-mode in target domain, we learn the generic translation mapping from the source domain to target domain, then impose such translation mapping upon a new source-mode graph with totally different topology (e.g., transportation network in another city) in order to synthesize the potential graphs under target-mode specific to it.

Similar to the issues we meet in image and text translation problems, however, usually the pairs of source-mode graph and targetmode graph are not available. For example, it is usually difficult or prohibitive to find a pair of normal transportation network and the post-snow-storm network with all their confounding factors the same such as the time, the weekday, the social events in the city which all can influence the traffic patterns. Therefore, it is impossible to distill the true portion of difference between normalmode and target-mode that is purely triggered by the snow storm instead of any other factors. In this paper, for the first time, we attack the unpaired graph-to-graph translation problem where we learn the graph translation mapping from source domain to target domain without pairwise graphs of both source and target modes. As shown in Figure 1, due to the absence of pairwise correlation, we need to learn the translation on the set of source-mode graphs to the set of target-mode graphs, instead of learning the pairwise mapping from one source-mode graph to the other target-mode graph. This is because we assume there is some underlying relationship between the domains: for example, that the traffic speed and flow under the same underlying road segments can be different in source-mode and target-mode, but for some other road segments, the difference is always not that large under various confounding. More concretely, we exploit supervision at the level of sets: we are given one set of graph in source domain $X$ and a different set in target domain $Y$. We may train a mapping $G: X \rightarrow Y$ such that the output $\hat{y}=G(x), x \in X$, is indistinguishable from graphs $y \in Y$ by an adversary trained to classify $\hat{y}$ apart from $y$. Such adversary classifier is conditioned on the input graph $x$. Different from learning the translation from image to image, $G$ is to translate from a graph to another graph, which will leverage the most recent techniques on graph translator [17], consisting of three parts: 1) graph convolutional layers, 2) graph deconvolutional layers, and 3) a graph U-net. Finally, this objective can induce an output distribution over $\hat{y}$ that matches the empirical distribution pdata(y) [47]. The optimal $G$ thereby translates the source domain $X$ to a target domain $\hat{Y}$ distributed identically to $Y$.

However, analogical to unpaired image-to-image translation [47], such a translation does not guarantee that an individual input $x$ and output $y$ are paired up in a meaningful way: there are infinitely many mappings $G$ that will induce the same distribution over $\hat{y}$. To address this problem, we propose Unpaired Graph TranslationGenerative Adversarial Nets (UGT-GAN) by extending the cycle consistency loss [47] previously designed for unpaired image-toimage translation and generalizing it into graph cycle consistency loss for unpaired graph translation. Specifically, graph cycle consistency adds an opposite direction translation from target to source domain: $F: Y \rightarrow X$. By training both the mapping $G$ and $F$ simultaneously, and adding a cycle consistency loss that encourages $F(G(x)) \approx x$ and $G(F(y)) \approx y$. Combining this loss with adversarial losses on domains $X$ and $Y$ yields our full objective for unpaired graph translation. The major contributions of this paper is as follows:

- Propose a new deep-learning framework for local event synthesis in networks. This paper proposes to synthesize events in specific locations especially for those without any historical event records, by transferring the generic knowledge from historical event occurrences in other local networks. The framework is generic, domain-independent, endto-end, and data-driven, without requiring intensive domain knowledge.

- Develop a new method for learning graph translation without pairwise samples. Synthesizing local events requires to translate the source-mode graphs without events into the target-mode graphs impacted by the events. In order to learn such translation without intensive human labors for labeling, we propose a new unpaired graph-to-graph translation model by leveraging conditional graph generative adversarial nets and cycle consistency loss.

- Conduct experiments in both synthetic and real-world datasets. The proposed method is compared with the stateof-the-art graph translation method. The analyses of the effectiveness in different metrics as well as the case studies of generated graphs demonstrate the advantages and good potential of the proposed method.

The rest of this paper is organized as follows. Section 2 reviews the background and related work while Section 3 introduces the model including problem setup, model formulation, and implementations. Section 4 presents the experiments on synthetic and real-world datasets. And the paper concludes with a summary of the research in Section 5. 


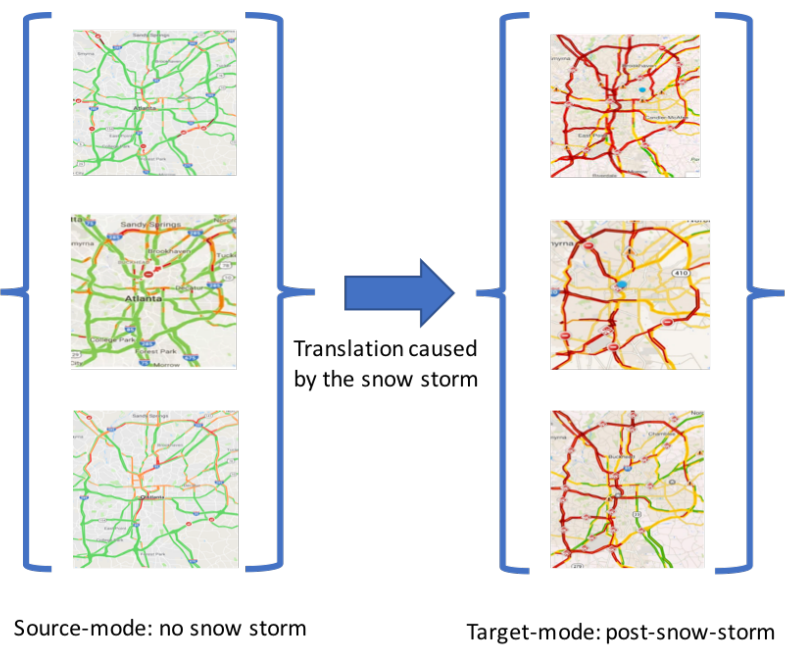

Figure 1: The transportation network before (left) and after (right) snow storm in a location. The warmer the color is for a road segment, the slower the traffic flow is inside it.

\section{RELATED WORK}

Deep graph representation learning. Recently a lot of research has been conducted in graph deep learning field. Generally specking there are two main directions: Graph Recurrent Networks $[15,27,38]$ and Graph Convolutional Networks [5, 9, 21, 24, 32$34,45]$. Early works for graph recurrent networks such as Gori et al. [15] were based on recursive neural networks. Later on more advanced recurrent network structures such as gated recurrent networks [27] was introduced in graph field. Another line of research is to generalize convolutional neural networks from grids (e.g., images) to generic graphs. Spectral graph convolutional neural networks was proposed by Bruna et al. [3]. Defferrard et al. [9] followed up this direction and extended the model using fast localized convolutions. Later on Kipf et al. [24] further introduced a fast approximation for a more efficient model. For most of the existing works, the graph topology is fixed, the nodes, edges and weights are all the same for all the graph samples. In order to take graph topology into account, Kwahara et al. [21] presented a new graph convolutional architecture that consists of a sequence of new layers for the convolution operations on edge-to-edge, edge-to-node, and node-to-graph for undirected graphs.

Graph generation. Graph generation is attracting much attention and it has many important applications in both mathematics and physics, however current graph generation methods are still limited. Some of the existing works followed the direction in domain specific graph generation $[8,13,20,26]$, which typically take sequence inputs and generate another sequences or parse trees from context-free grammars or sub-graphs by utilizing the collection of valid components, by leveraging the celebrated Seq2Seq model [41] based on variational autoencoder (VAE) [23]. These methods typically are limited in specific domain. Few generic graph generation models have been proposed recently based on VAE [37, 40] and generative adversarial nets (GAN) [2] and others [29]. Specifically, Li et al. [29] proposed a graph net that generates nodes and edges sequentially to form a whole graph, which is sensitive to the generation order and time consuming for large graph. Bojchevski et al. [2] proposed a random walk based model which also faces similar problems. Simonovsky et al. [40] and Samanta et al. [37] both propose new VAE models for whole graph generation, but the model cannot scale well in terms of both memory and runtime. Guo et al. [17] has tackled the paired graph translation problems and have some interesting results. However, paired translation need to have explicit mapping between source graph and target graph. Unpaired translation is more challenging since it requires the model to learn the distribution of realistic graphs and thus be able to learn translation rules implicitly.

Conditional generative adversarial nets. In image generation domain, Generative adversarial networks (GANs) [14] has greatly advanced the state of the art. Many follow up works have further empowered GANs to generate objects under certain conditions [31, 36, 44]. Furthermore, conditional GANs has enabled multi-modal data fusion to generate images according to specific annotations and even making transfer between images. Many important applications in image generation domain including imageto-image translation [19] and style transfer [47] has been proposed. However, currently, to the best of our knowledge, there is little to no work focusing on unpaired graph-to-graph translation problem.

\section{MODEL}

We first present the problem formulation of unpaired graph translation. We will then move on to propose our method UGT-GAN and describe the framework in details.

\subsection{Problem Setup}

The paper focuses on unpaired graph translation, which can relate graphs from two domains, namely source domain and target domain, without explicit pairing or mapping between each graph of two domains. Here we define the source domain $X=(V, E, A)$ as a directed weighted graph such that $V$ is the set of $N$ nodes, $E \subseteq V \times V$ is the set of directed edges, and $A \in \mathbb{R}^{n \times n}$ is a matrix holding the set of weights for the corresponding edges, called weighted adjacency matrix. Denote $e_{i, j} \in E$ as an edge from the node $v_{i} \in V$ to $v_{j} \in V$ and thus $w_{i, j} \in W$ is used to denote the corresponding weight of the edge $e_{i, j}$. Similarly, we define the target domain $Y=\left(V^{\prime}, E^{\prime}, A^{\prime}\right)$ also as directed weighted graph. Typically we focus on learning the translation from one topological patterns to the other one the same set of nodes and hence we have $V=V^{\prime}$. Additionally, the weights of edges are nonnegative such that $A_{i, j} \geq 0$ and $A_{i, j}^{\prime} \geq 0$. We define the problem of unpaired graph translation as learning the translation rules between two domains as $G: X \rightarrow Y$ and $F: Y \rightarrow X$.

\subsection{Model Formulation}

Given training samples of graphs $x \in X$ and $y \in Y$ from the two domains, we denote the data distribution as $x \sim \operatorname{pdata}(x)$ and $y \sim \operatorname{pdata}(y)$, respectively. As illustrated in Figure 2, our model includes two mappings $G: X \rightarrow Y$ and $F: Y \rightarrow X$. In addition, we introduce two adversarial graph discriminators $D_{X}$ and $D_{Y}$, where $D_{X}$ aims to distinguish between real graphs $x$ and translated graphs $F(y)$; similarly, $D_{Y}$ aims to discriminate between $y$ and $G(x)$. Our objective contains two types of terms: adversarial losses 


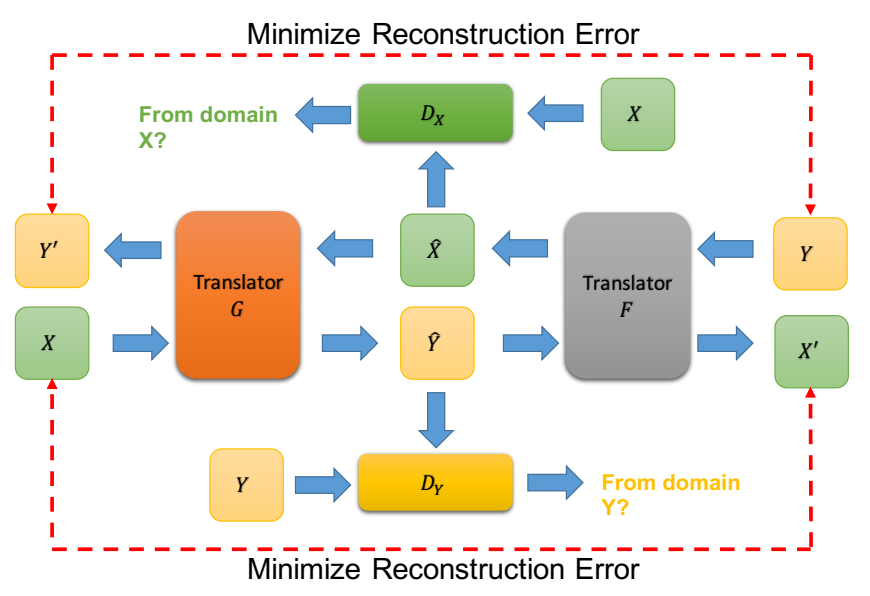

Figure 2: The flowchart of UGT-GAN. Our model consists of two graph translators $G$ and $F$ for learning the graph mapping functions $G: X \rightarrow Y$ and $F: Y \rightarrow X$, and two associated adversarial graph discriminators $D_{Y}$ and $D_{X} \cdot D_{Y}$ encourages $G$ to translate domain $X$ graphs to be similar with domain $Y$, and vice versa for $D_{X}$. In addition, two cycle consistency losses $X \rightarrow G(X) \rightarrow F(G(X)) \approx X$ and $Y \rightarrow F(Y) \rightarrow G(F(Y)) \approx Y$ are introduced to further regularize the translation rules learned.

[14] for matching the distribution of generated graphs to the data distribution in the target domain; and graph cycle consistency losses to prevent the learned mappings $G$ and $F$ from contradicting each other.

\subsection{Cycle Consistency in Graphs}

Although adversarial training can theoretically learn mappings $G$ and $F$ that yield same distribution as target domains graph $Y$ and $X$ respectively. The adversarial losses alone cannot guarantee that the learned function can map an individual source graph to a desired target graph. Therefore, in order to regularize the mapping functions in the model, we introduce the cycle consistency in the mapping functions $G$ and $F$ that: for each graph from domain $X$, the model with translation cycle $X \rightarrow G(x) \rightarrow F(G(x))$ result should be approximately same with original source graph $x$, as shown in Figure 1. Similarity, translation cycle $y \rightarrow G(y) \rightarrow F(G(y))$ result should be approximately same with original target graph $y$. Now we propose the regularization term that can constraint the model to have cycle consistency behavior as:

$$
\mathbb{E}_{x \sim \text { pdata }(x)}\left[\|F(G(x))-x\|_{2}\right]+\mathbb{E}_{y \sim \text { pdata }(y)}\left[\|F(G(y))-y\|_{2}\right]
$$

\subsection{UGT-GAN}

Now, combining the above regularization term with adversarial losses, we propose our Unpaired Graph Translation Generative Adversarial Networks (UGT-GAN) model objective as:

$$
\begin{gathered}
\mathcal{L}=\mathbb{E}_{y \sim \text { pdata }(y)}\left[\log D_{Y}(y)\right]+\mathbb{E}_{X \sim \text { pdata }(x)}\left[\log \left(1-D_{Y}(G(x))\right)\right] \\
+\mathbb{E}_{x \sim \text { pdata }(x)}\left[\log D_{X}(x)\right]+\mathbb{E}_{y \sim \text { pdata }(y)}\left[\log \left(1-D_{X}(F(y))\right)\right](2) \\
+\lambda\left(\mathbb{E}_{x \sim \text { pdata }(x)}\left[\|F(G(x))-x\|_{2}\right]+\mathbb{E}_{y \sim \text { data }(y)}\left[\|F(G(y))-y\|_{2}\right]\right)
\end{gathered}
$$

where $D_{Y}$ and $D_{x}$ are trained to discriminate between model translated graphs and real sample graphs in domain $Y$ and $X$ respectively; functions $G$ and $F$ try to fool discriminator $D_{Y}$ and $D_{X}$ by generating realistic like graphs; the scaling factor $\lambda$ controls the relative importance of regularization term on restrict the model to follow cycle consistent constraint. The problem we are solving in the above formula is:

$$
G^{*}, F^{*}=\underset{G, F}{\operatorname{argmin}} \max _{D_{X}, D_{Y}} \mathcal{L}
$$

\subsection{Implementation}

For generative adversarial network architecture, we adopt from Guo et al. [17] who have shown impressive results for paired graph translation. The network contains several graph convolution on discriminator and both graph convolution and deconvolution layers on the translator in order to output generated graphs. In addition, several additional tricks are used, such as "U" shape network structure, which can improve the performance. In order to stabilize training process of UGT-GAN, we replace the original negative log likelihood objective by a least-squares loss [30]. For all the experiments, $\lambda$ is set to $10^{-5}$ in equation. We used the Adam solver [22] for model optimization.

\section{EXPERIMENTS}

In this section, extensive experiments on unpaired graph translation for the proposed methods on synthetic and real-world graph datasets are conducted and analyzed. The proposed UGT-GAN demonstrated outstanding performance in both indirect and direct evaluation metrics. All experiments are conducted on a 64-bit machine with Navida GPU (GTX 1070,1683 MHz, 8 GB GDDR5).

\subsection{Experimental Settings}

Poisson-random Graph Dataset. In order to validate the performance of the proposed UGT-GAN, a synthetic dataset based on Poisson-random graphs is used. The graphs size (number of nodes) is set to 100 . The dataset consists total of 5000 samples of source and target graphs: 2000 are used for training the graph translator and the remaining are used as test set to validate. The source-mode graph is generated by Barabasi-Albert model [1] which is a scalefree network. Then for a source-mode graph with number of $|E|$ edges, we randomly add another $k \cdot|E|$ edges on it to form the target-mode graph, where $k$ follows the Poisson distribution with mean of 5 .

U.S. Influenza Outbreak Keyword Co-occurrence Dataset.. The dataset for influenza outbreaks in the U.S. use Twitter data as the data source. A total number of 1,266,301 tweets that contain the flu-related keywords like 'flu' and 'influenza' are included in the dataset. We formulated the tweet data into keyword co-occurrence graphs using a keyword list of size 100 , where each node is a keyword and each edge is the frequency of the co-occurrence between 
Table 1: Importance of keyword co-occurrence correlation and networks when identifying from tweet on whether its author reports the infection of himself/herself. Individual keywords do not have good discriminative power. Whether "flu" indicates "positive", "neutral", or "negative" depends heavily on the keyword(s) it co-occurs with.

\begin{tabular}{l|l|l|l}
\hline ID & tweet & keyword co-occurrence & flu infection? \\
\hline 1 & I feel like crap! I'm lying in the bed and getting the flu & bed, flu & positive \\
2 & For best protection from flu, I've just got shot & flu, shot & negative \\
3 & This flu is just trolling me now, staying home today & flu, home & positive \\
4 & CDC confirms 11 flu as influenza A: https://... & flu, influenza, <hyperlink> & neutral \\
5 & Just got my flu shot from @rxjude ouch!! & flu, shot & negative \\
6 & Science may just ... to treat the flu http://wef.ch/2... & flu, <hyperlink> & neutral \\
7 & If a cop ever shot my dog then I'm gettin shot too & shot & neutral \\
\hline
\end{tabular}

Table 2: Evaluation for Poisson random graphs

\begin{tabular}{ccccc}
\hline Model & Precision & Recall & AUC & F1 \\
\hline GT-GAN & 0.90 & 1.00 & 1.00 & 0.94 \\
UGT-GAN & 0.93 & 0.99 & 1.00 & 0.98 \\
Gold Standard & 1.00 & 0.99 & 1.00 & 0.99 \\
\hline
\end{tabular}

two keywords. The keyword list contains disease related words like "flu", "fever", "cough" as well as general words like "bed", "home", "work". When detecting and forecasting future influenza outbreaks using social media data it is important and fundamental to accurately identify the future potential amount of infectious people. And the keyword co-occurrence patterns and graphs is highly important for the inference of influenza symptoms and infections of individuals. As shown in Table 1, for example, when "flu" occurs with words like "home" and "bed" indicates the author's flu infection and isolation, while it is mentioned together with "shot" then it is about "vaccination", but when a hyperlink co-occurs with it then it may be just a news. The influenza outbreaks have been labeled by reports from the Centers for Disease Control and Prevention (CDC) [6]. CDC publishes the weekly influenza-like illness (ILI) activity level for each state in the U.S. based on the proportional level of outpatient visits to healthcare providers for ILI. There is a flu outbreak if the ILI activity level is 'high', otherwise there is no flu outbreak. Here the source-mode graphs mean the keyword co-occurrence graphs for a day corresponding to no flu outbreaks while target-mode corresponds to the graphs for a day under flu outbreaks. The graph samples from the year of 2011 is used for training and samples of the year of 2012 is used for performance evaluation.

\subsubsection{Evaluation Metrics.}

Direct Evaluation To directly verify if UGT-GAN indeed can discover the underlying ground-truth translation rules between input and target graphs, we examined the degree distribution and the ratio of added edges for poisson-random graph dataset. For Poissonrandom graph, the distributions of $k$ in the real target graphs and those generated graphs are compared.

For the flu outbreak dataset, the real target graphs and those generated by UGT-GAN and comparison method are compared under well-recognized graph metrics including degree of nodes, reciprocity, and density. We calculate the distance of degree distribution and Mean Squared Error (MSE) for reciprocity and density.
Table 3: Squared difference of Graph properties measurements for influenza outbreak keyword co-occurrence graph

\begin{tabular}{cccc}
\hline Model & Density & Average Degree & Reciprocity \\
\hline GT-GAN & 0.0792 & 7.0766 & 1.0000 \\
UGT-GAN & 0.0687 & 6.7780 & 0.0107 \\
\hline
\end{tabular}

Indirect Evaluation One important goal of graph translation is to generate the target graph for any new-coming source graph. Take our real-world dataset as an instance, flu outbreak network can be used to forecast whether future flu activities will outbreak. However, historical flu outbreak sample is rare. This can be handled by graph translator which learns the translation mapping to translate a normal flu activity graph into an flu-outbreak graph. Thus, we use the generated graphs to train a classifier, and if this classifier can classify accurately on real input and target graphs, it demonstrates the effectiveness and high fidelity of the generated graphs. Specifically, first, we train the graph translator based on training set and generate the target graphs based on the source graphs in test set. Then, we split the test set into two parts. First part is used to train graph classifiers that distinguish source graphs from target graphs. The classifier is trained by generated target graphs by the comparison methods. In addition, we also train another "gold standard" classifier based on the real target graphs. Then the second part test data is used to test and compare the classification performance against the real target graphs in this part. Therefore, if the performance of the classifier trained by the graphs generated by a comparison method is good and close to the gold-standard one, it demonstrates the effectiveness of this comparison method.

4.1.2 Comparison Methods.

There is no existing work on unpaired graph translation, thus we will try to compare with paired graph translation model GT-GAN [17] as baseline method. For the network structure of GT-GAN, we followed the same settings based on their paper. There is one hyperparameter $\lambda$ in GT-GAN, which is the scaling factor that controls the paired regularization constraints. We set $\lambda$ to 0 as no pairing information is given in our unpaired graph translation dataset.

\subsection{Performance}

4.2.1 Performance on Poisson Random Graph Set.

Graphs are generated by UGT-GAN and GT-GAN on test set. The node degree distributions of the generated graphs are compared 
Table 4: Classification performance on U.S. influenza outbreak keyword co-occurrence graph

\begin{tabular}{ccccc}
\hline Model & Precision & Recall & AUC & F1 \\
\hline GT-GAN & 0.90 & 0.07 & 0.48 & 0.13 \\
UGT-GAN & 0.76 & 0.10 & 0.57 & 0.18 \\
Gold Standard & 0.70 & 0.55 & 0.73 & 0.61 \\
\hline
\end{tabular}

with those of real target graphs in several distances metrics, as shown in Table.

Direct evaluation The mean of edge increasing ratio $k$ for generated graphs by our UGT-GAN is 3.8, compared to the real value of 5, which implies that the UGT-GAN generally is able to discover the underlying increasing ratio between input and target graphs.

Indirect Evaluation The precision, recall, AUC, and F1-measure of three classifiers are listed in the Table 2. UGT-GAN exhibits outstanding performance and is highly close to the "gold standard". This indicates that UGT-GAN can effectively learn the rule to translate the source graph to target graph without explicit pairing.

\subsubsection{Performance on influenza outbreak keyword co-occurrence graph.}

Direct evaluation We use three metrics to evaluate the similarity of generated graphs and real graphs. The squared difference are calculated to measure the similarity between real and generated graphs on average. Table 3 shows that UGT-GAN enjoys less divergence in terms of density and reciprocity comparing with GT-GAN This may suggest that with cycle consistent regularization UGTGAN is able to learn the target graph distribution overall thus yields generated graphs closer to realistic Target graphs.

In addition, we conduct a statistical analysis on the average degree of each keyword among input, translated and Target graphs in flu outbreak dataset, as shown in Figure 3. We can see regular flu activity graphs has much less degree on average among all the keywords comparing with target outbreak graphs. The green bar in the middle shows that our proposed model can indeed learn the translation rules and successfully increased the degree of each keyword to approximately match with the ground truth target graphs.

Indirect Evaluation For U.S. influenza outbreak dataset, our goal is to train a classifier that can forecast whether there will be a flu outbreak in the near future based on the flu related keyword co-occurrence graph. UGT-GAN is trained by the samples from half of training data and then generate flu outbreak graph samples accordingly. Then the generated graphs are used as positive samples to train a classifier to show whether the model indeed capture the translation rule of source and target graphs. As shown in Table 4, classifier trained by UGT-GAN can indeed capture some correlation of regular graphs and flu outbreak graphs. UGT-GAN gets the AUC of 0.57, while without explicit pairing, GT-GAN failed to learn anything interesting and thus yields a random like model with AUC of 0.5. UGT-GAN significantly outperforms GT-GAN on all the metrics.

\subsection{Case Study : Flu Keyword Co-occurrence Graph Translation}

In the previous section we have demonstrated the effectiveness of UGT-GAN model on translating regular graph to flu outbreak graph. Now we will conduct a case study that analyze and visualize the model generated keyword graphs in order to get qualitative understanding of the generated graphs.

Figure 4 shows the example of influenza outbreak keyword cooccurrence graphs with regular flu activity graph, flu outbreak graph translated from regular graph by UGT-GAN, and realistic flu outbreak graph from left to right. Each node is labeled with the corresponding keyword; the thickness of the edge represents relative edge weight, the thicker the edge the higher the edge weight. Here only the largest connect subgraph is displayed for simplicity. We can observe that, during the regular day when flu activity is low, the keyword co-occurrence graph is sparse, this is easy to understand because less people are talking about flu related words during regular day. However, we can still observe some typical characteristics of the graph: for example, keyword 'flu' seems to be the central node or hub node for the graph; keywords 'sick', 'stomach' and 'feeling' also have strong correlation with flu with thick edges. However, those keywords may just indicate common cold symptom, thus typically are not good indicator to distinguish between common cold with severe influenza outbreak. However, when the influenza indeed outbreak, as shown on the right graph, more flu related keywords appear in the subgraph. Keywords such as 'meds', 'die' and 'death' indicate more severe situation during disease outbreak; in addition, keywords that related to time such as 'morning', 'season' and 'weekend' appear in the graph which may indicate the duration and time period of the outbreak. Interestingly, those indicators also appear in our model translated graph. We observe keywords 'meds', 'hospital' and 'die' that indicate serve situation of flu outbreak; keywords 'days', 'week', 'weeks' and 'weekend' that correlated with flu outbreak duration. Surprisingly, our model even captured keywords 'ebola', 'swine', 'pigs' and 'avian' that directly indicate which kind of influenza is ongoing. Those keywords can be crucial discriminative features for the classifier to distinguish regular flu activity graph and flu outbreak graph. This observation clearly demonstrates that our model indeed found meaningful translation rules from regular flu activity to flu outbreak graph.

\section{CONCLUSIONS}

In this work, we propose an approach for translation of graphs from their source-mode to target-mode such that the generated targetmode is indistinguishable from the distribution of the real targetmode using an adversarial loss. We also learn a inverse translation from target-mode to source-mode and couple these two translation mappings through cycle consistency loss. Extensive experiments on both synthetic and real-world application data demonstrate that the proposed approaches is capable of generating graphs close to real target graphs. Case studies on the synthesized networks have also been illustrated and analyzed to show the reasonableness of the generated target-mode graphs. 


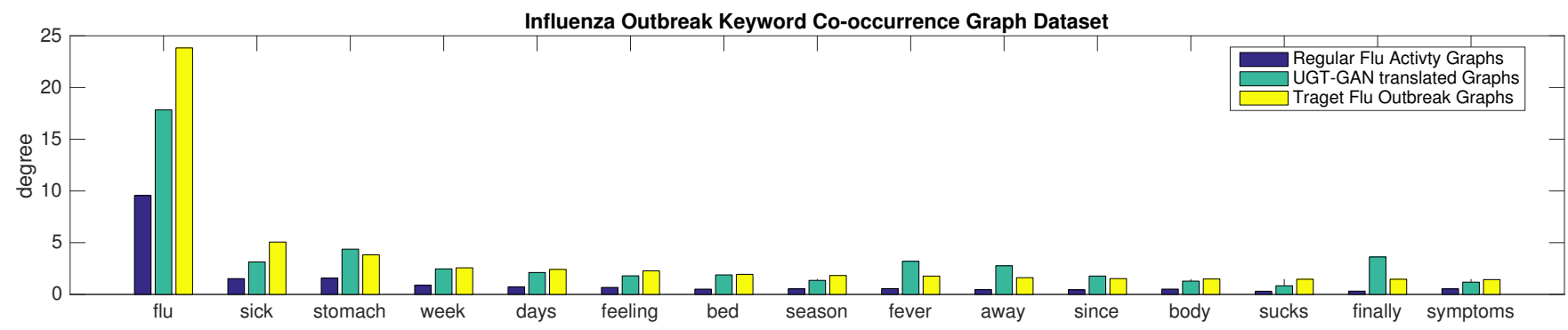

Figure 3: Comparison of the average node degree of 15 most frequent keywords among input, translated and target graphs in flu outbreak dataset.
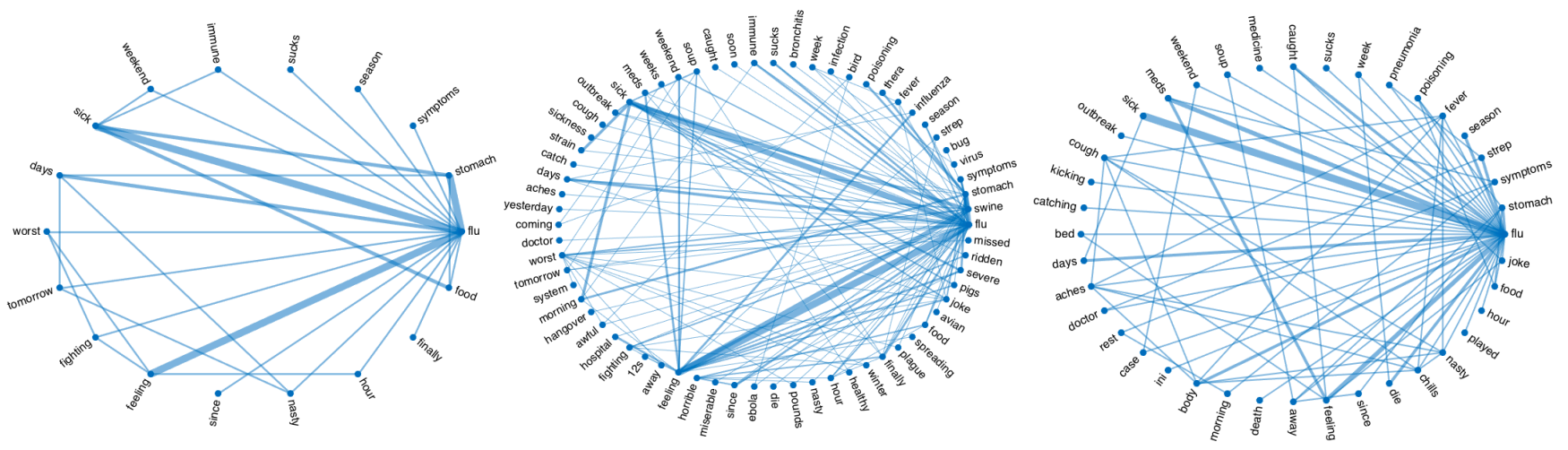

Figure 4: Comparison of input graph (left), model translated graph (middle), and target flu outbreak graph (right).

\section{ACKNOWLEDGEMENT}

This work was supported by the National Science Foundation grant: $\# 1755850$.

\section{REFERENCES}

[1] Réka Albert and Albert-László Barabási. 2002. Statistical mechanics of complex networks. Reviews of modern physics 74, 1 (2002), 47.

[2] Aleksandar Bojchevski, Oleksandr Shchur, Daniel Zügner, and Stephan Günnemann. 2018. NetGAN: Generating Graphs via Random Walks. arXiv preprint arXiv:1803.00816 (2018)

[3] Joan Bruna, Wojciech Zaremba, Arthur Szlam, and Yann LeCun. 2013. Spectral networks and locally connected networks on graphs. arXiv preprint arXiv:1312.6203 (2013).

[4] Joan Bruna, Wojciech Zaremba, Arthur Szlam, and Yann Lecun. 2014. Spectral networks and locally connected networks on graphs. In nternational Conference on Learning Representations (ICLR2014), CBLS, April 2014.

[5] Shaosheng Cao, Wei Lu, and Qiongkai Xu. 2016. Deep Neural Networks for Learning Graph Representations.. In AAAI. 1145-1152.

[6] CDC. [n. d.]. https://www.cdc.gov/.

[7] Feng Chen, Baojian Zhou, Adil Alim, and Liang Zhao. 2017. A Generic Framework for Interesting Subspace Cluster Detection in Multi-attributed Networks. In Data Mining (ICDM), 2017 IEEE International Conference on. IEEE, 41-50.

[8] Hanjun Dai, Yingtao Tian, Bo Dai, Steven Skiena, and Le Song. 2018 Syntax-Directed Variational Autoencoder for Structured Data. arXiv preprint arXiv:1802.08786 (2018).

[9] Michaël Defferrard, Xavier Bresson, and Pierre Vandergheynst. 2016. Convolutional neural networks on graphs with fast localized spectral filtering. In Advances in Neural Information Processing Systems. 3844-3852.

[10] Paul Erdos and Alfréd Rényi. 1960. On the evolution of random graphs. Publ. Math. Inst. Hung. Acad. Sci 5, 1 (1960), 17-60.

[11] N Garner. 2014. In Atlanta snow storm, itâĂŹs social media to the rescue. Retrieved 3rd fune (2014).

[12] Justin Gilmer, Samuel S Schoenholz, Patrick F Riley, Oriol Vinyals, and George E Dahl. 2017. Neural message passing for quantum chemistry. arXiv preprint arXiv:1704.01212 (2017).

[13] Rafael Gómez-Bombarelli, Jennifer N Wei, David Duvenaud, José Miguel Hernández-Lobato, Benjamín Sánchez-Lengeling, Dennis Sheberla, Jorge
Aguilera-Iparraguirre, Timothy D Hirzel, Ryan P Adams, and Alán Aspuru-Guzik. 2018. Automatic chemical design using a data-driven continuous representation of molecules. ACS central science 4, 2 (2018), 268-276.

[14] Ian Goodfellow, Jean Pouget-Abadie, Mehdi Mirza, Bing Xu, David Warde-Farley, Sherjil Ozair, Aaron Courville, and Yoshua Bengio. 2014. Generative adversarial nets. In Advances in neural information processing systems. 2672-2680.

[15] Marco Gori, Gabriele Monfardini, and Franco Scarselli. 2005. A new model for learning in graph domains. In Neural Networks, 2005. IfCNN'05. Proceedings. 2005 IEEE International foint Conference on, Vol. 2. IEEE, 729-734.

[16] Aditya Grover and Jure Leskovec. 2016. node2vec: Scalable feature learning for networks. In Proceedings of the 22nd ACM SIGKDD international conference on Knowledge discovery and data mining. ACM, 855-864.

[17] Xiaojie Guo, Lingfei Wu, and Liang Zhao. 2018. Deep Graph Translation. arXiv preprint arXiv:1805.09980 (2018).

[18] Will Hamilton, Zhitao Ying, and Jure Leskovec. 2017. Inductive representation learning on large graphs. In Advances in Neural Information Processing Systems. $1025-1035$.

[19] Phillip Isola, Jun-Yan Zhu, Tinghui Zhou, and Alexei A Efros. 2017. Image-toimage translation with conditional adversarial networks. arXiv preprint (2017).

[20] Wengong Jin, Regina Barzilay, and Tommi Jaakkola. 2018. Junction Tree Variational Autoencoder for Molecular Graph Generation. arXiv preprint arXiv:1802.04364 (2018).

[21] Jeremy Kawahara, Colin J Brown, Steven P Miller, Brian G Booth, Vann Chau, Ruth E Grunau, Jill G Zwicker, and Ghassan Hamarneh. 2017. BrainNetCNN: convolutional neural networks for brain networks; towards predicting neurodevelopment. NeuroImage 146 (2017), 1038-1049.

[22] Diederik P Kingma and Jimmy Ba. 2014. Adam: A method for stochastic optimization. arXiv preprint arXiv:1412.6980 (2014)

[23] Diederik P Kingma and Max Welling. 2013. Auto-encoding variational bayes. arXiv preprint arXiv:1312.6114 (2013).

[24] Thomas N Kipf and Max Welling. 2016. Semi-supervised classification with graph convolutional networks. arXiv preprint arXiv:1609.02907 (2016).

[25] Thomas N. Kipf and Max Welling. 2017. Semi-Supervised Classification with Graph Convolutional Networks. In International Conference on Learning Representations (ICLR).

[26] Matt J Kusner, Brooks Paige, and José Miguel Hernández-Lobato. 2017. Grammar variational autoencoder. arXiv preprint arXiv:1703.01925 (2017).

[27] Yujia Li, Daniel Tarlow, Marc Brockschmidt, and Richard Zemel. 2015. Gated graph sequence neural networks. arXiv preprint arXiv:1511.05493 (2015). 
[28] Yujia Li, Daniel Tarlow, Marc Brockschmidt, and Richard Zemel. 2016. Gated graph sequence neural networks. International Conference on Learning Representations (2016).

[29] Yujia Li, Oriol Vinyals, Chris Dyer, Razvan Pascanu, and Peter Battaglia. 2018. Learning deep generative models of graphs. arXiv preprint arXiv:1803.03324 (2018).

[30] Xudong Mao, Qing Li, Haoran Xie, Raymond YK Lau, Zhen Wang, and Stephen Paul Smolley. 2017. Least squares generative adversarial networks. In Computer Vision (ICCV), 2017 IEEE International Conference on. IEEE, 2813-2821.

[31] Mehdi Mirza and Simon Osindero. 2014. Conditional generative adversarial nets. arXiv preprint arXiv:1411.1784 (2014).

[32] Seyedeh Fatemeh Mousavi, Mehran Safayani, Abdolreza Mirzaei, and Hoda Bahonar. 2017. Hierarchical graph embedding in vector space by graph pyramid. Pattern Recognition 61 (2017), 245-254.

[33] Mathias Niepert, Mohamed Ahmed, and Konstantin Kutzkov. 2016. Learning convolutional neural networks for graphs. In International conference on machine learning. 2014-2023.

[34] Giannis Nikolentzos, Polykarpos Meladianos, Antoine Jean-Pierre Tixier, Konstantinos Skianis, and Michalis Vazirgiannis. 2017. Kernel Graph Convolutional Neural Networks. arXiv preprint arXiv:1710.10689 (2017).

[35] Bryan Perozzi, Rami Al-Rfou, and Steven Skiena. 2014. Deepwalk: Online learning of social representations. In Proceedings of the 20th ACM SIGKDD international conference on Knowledge discovery and data mining. ACM, 701-710.

[36] Scott Reed, Zeynep Akata, Xinchen Yan, Lajanugen Logeswaran, Bernt Schiele and Honglak Lee. 2016. Generative adversarial text to image synthesis. arXiv preprint arXiv:1605.05396 (2016).

[37] Bidisha Samanta, Abir De, Niloy Ganguly, and Manuel Gomez-Rodriguez. 2018 Designing random graph models using variational autoencoders with applications to chemical design. arXiv preprint arXiv:1802.05283 (2018).

[38] Franco Scarselli, Marco Gori, Ah Chung Tsoi, Markus Hagenbuchner, and Gabriele Monfardini. 2009. The graph neural network model. IEEE Transactions on Neural Networks 20, 1 (2009), 61-80.

[39] Shashi Shekhar, Zhe Jiang, Reem Y Ali, Emre Eftelioglu, Xun Tang, Venkata Gunturi, and Xun Zhou. 2015. Spatiotemporal data mining: a computational perspective. ISPRS International fournal of Geo-Information 4, 4 (2015), 2306-2338.

[40] Martin Simonovsky and Nikos Komodakis. 2018. GraphVAE: Towards Generation of Small Graphs Using Variational Autoencoders. arXiv preprint arXiv:1802.03480 (2018).

[41] Ilya Sutskever, Oriol Vinyals, and Quoc V Le. 2014. Sequence to sequence learning with neural networks. In Advances in neural information processing systems. 31043112.

[42] Jian Tang, Meng Qu, Mingzhe Wang, Ming Zhang, Jun Yan, and Qiaozhu Mei. 2015. Line: Large-scale information network embedding. In Proceedings of the 24th International Conference on World Wide Web. International World Wide Web Conferences Steering Committee, 1067-1077.

[43] Xiao Wang, Peng Cui, Jing Wang, Jian Pei, Wenwu Zhu, and Shiqiang Yang. 2017 Community Preserving Network Embedding.. In AAAI. 203-209.

[44] Xiaolong Wang and Abhinav Gupta. 2016. Generative image modeling using style and structure adversarial networks. In European Conference on Computer Vision. Springer, 318-335.

[45] Kun Xu, Lingfei Wu, Zhiguo Wang, and Vadim Sheinin. 2018. Graph2Seq: Graph to Sequence Learning with Attention-based Neural Networks. arXiv preprint arXiv:1804.00823 (2018)

[46] Liang Zhao, Qian Sun, Jieping Ye, Feng Chen, Chang-Tien Lu, and Naren Ramakrishnan. 2015. Multi-task learning for spatio-temporal event forecasting. In Proceedings of the 21th ACM SIGKDD International Conference on Knowledge Discovery and Data Mining. ACM, 1503-1512.

[47] Jun-Yan Zhu, Taesung Park, Phillip Isola, and Alexei A Efros. 2017. Unpaired image-to-image translation using cycle-consistent adversarial networks. arXiv preprint (2017) 